\title{
Asymmetric Trimmed Median Filter for Images Highly Corrupted with Random valued Impulse Noise
}

\author{
R.Pushpavalli \\ Electronics and Communication Engineering \\ Pondicherry Engineering College \\ Puducherry, \\ India-605 014
}

\author{
G.Sivaradje \\ Electronics and Communication Engineering \\ Pondicherry Engineering College \\ Puducherry, \\ India-605 014
}

\begin{abstract}
Asymmetric Trimmed Median Filter for Image denoising is proposed in this paper. This technique can be used for restoring the images extremely corrupted with random valued impulse noise. This paper introduces an impulse detection technique and decision based median filter for restoring the corrupted images. The detection technique is used for discriminating between corrupted and uncorrupted image pixels. The corrupted pixels are restored using Asymmetric trimmed median filter. The performance of the proposed restoring scheme is evaluated with random valued impulse noise for different test images. Simulation results show that this method is significantly better than a number of existing techniques in terms of image restoration and noise detection.
\end{abstract}

\section{Keywords}

Impulse noise, Median filters, Image processing, Restoration.

\section{INTRODUCTION}

Digital images are often corrupted by impulse noise due to transmission errors, malfunctioning pixel elements in the camera sensors, faulty memory locations, and timing errors in analog-to-digital conversion. In most applications, denoising the image is fundamental to subsequent image processing operations, such as edge detection, image segmentation, object recognition, etc [1-3]. The goal of noise removal is to suppress the noise while preserving image details. Removal of the impulse noise is done in two stages: detection of noisy pixel and replacement of that pixel. Median filter is used as a backbone for removal of impulse noise [4]. Many filters with an impulse detector have been proposed to remove impulse noise.

One of the most popular methods is the median filter, which can suppress noise with high computational efficiency. Since every pixel in the image is replaced by the median value in its neighborhood, the median filter often removes desirable details within the filtering window on the image and blurs it too. Several impulse noise removal methods with different kinds of noise detectors have been proposed in the literature, namely; switching based median filter, weighted median filter, center weighted median filter, Decision based median filters, etc [5-7]. The main drawback of these filters is that median values or their variations are used to restore the noisy pixels, and hence these median based filters are alters both noise free and noisy pixels. As a result, this performance will prone to misclassify the pixel's characteristics.

Some switching-based median filtering methodologies had been proposed for impulse noise elimination [8-22]. These filtering operations were obtained by applying "no filtering" to preserve true pixels and standard median filter to remove impulse noise. The median based filtering operations are crucial to achieve good filtering performance, especially at high noise density interference. Removal of random valued impulse noise algorithms have been developed [23-26]. Removal of random valued impulse noise from images without losing their features such as edges and fine details is difficult tasks in image filtering.

In order to address these issues, in this paper a powerful image restoring technique for highly corrupted images, namely Asymmetric trimmed median filter for images highly corrupted with random valued impulse noise is proposed. This filter is obtained in two stages; Noisy pixels of the corrupted image are identified using a Impulse Detection Technique in first stage. In this stage, the corrupted and uncorrupted pixels in the image are detected by checking the pixel element value against the dynamic range of high noise level (HNL) and low noise level (LNL) respectively. These values are the impulse noise intensity values. It is followed by an Asymmetric trimmed median filter (ATM) for recovering those corrupted pixels identified from IDT in the second stage. In an impulse detection stage, the current pixel is detected as an uncorrupted pixel and it is left unaltered, otherwise, it is corrupted. Then the proposed filtering technique is performed on it. The proposed filtering technique of impulse detection followed by ATM filter is capable of producing high quality images and it prevents image blurring compared to other denoising techniques. Extensive simulation experiments have been conducted to evaluate and determine the performance of the proposed filter in terms of quantitative and qualitative metrics.

The outline of this paper is as follows. In Section II, define the noise model. Section III describes noise detection and filtering technique in detail. Section IV gives simulation results to demonstrate the performance of the proposed filtering technique. Finally conclusions are drawn in section V.

\section{NOISE MODEL}

Impulse noise is caused by malfunctioning pixels in camera sensors, faulty memory locations in hardware, erroneous transmission in a channel. Generally two types of types of impulse nose namely Salt \& Pepper and random valued impulse noise [1] respectively. Salt \& pepper noise represents the pixel value of maximum (255) and minimum (0) intensity on digital images. Whereas, in the case of random valued impulse, digital images are often corrupted by any random value in the dynamic range of grayscale.

The proposed filter only detects random valued impulse noise present in digital images in very efficient manner then removes it. As the impulse noise is additive in nature, noise present in a region does not depend upon the intensities of pixels in that region. Based on the properties of probability, images are corrupted with impulse noise. The intensity of grayscale pixel 
is stored as an 8-bit integer with 256 possible different shades of gray going from black to white. It is represented as a gray scale range $[0, L-1]$ ( $L$ represents brightness value of 255) integer interval. In this paper the impulse valued noise is considered as random impulse noise. In case of images corrupted by this kind of random valued impulse noise, intensity of the pixel $X_{i j}$ at location (i,j) is described by the probability density function given by the following equation

$$
f\left(X_{i j}\right)= \begin{cases}p_{a} & \text { for } X_{i j}=a \\ 1-p & \text { for } X_{i j}=Y_{i j} \\ p_{b} & \text { for } X_{i j}=b\end{cases}
$$

where $a=1,2, \ldots m$ is the minimum intensity range (dark dot); $b=1,2, \ldots n$ is the maximum intensity range (light dot); $p_{a}$ is the probability of intensity generation $\mathrm{a} ; p_{b}$ is the probability of intensity generation a; $p$ is the noise density, and $Y_{i j}$ is the intensity of the pixel at location $(\mathrm{i}, \mathrm{j})$ in the uncorrupted image. If either pa or $p_{b}$ is zero the impulse noise is called unipolar noise. If neither probability is zero and especially if they are equal, impulse noise is called bipolar noise or salt-and-pepper noise. Malfunctioning CCD elements (i.e. hot and dead pixels) and flecks of dust inside the camera most commonly introduce the random valued impulse noise on digital images.

\section{IMPULSE DETECTION AND FILTERING TECHNIQUE}

\subsection{Impulse Detection Technique (IDT)}

The Impulse detection procedure is based on local measurements of impulse noise, which is referred to as impulse detection. An impulse detector realizes noise detection, which takes the output from the standard median filter and compares it with the original pixel value in order to make decisions. After decision mechanism, the filtering scheme decides whether the pixel is corrupted by impulse or not and then filtering is applied on it based on the decision of edge identifications and impulse detection is explained in Fig.1.

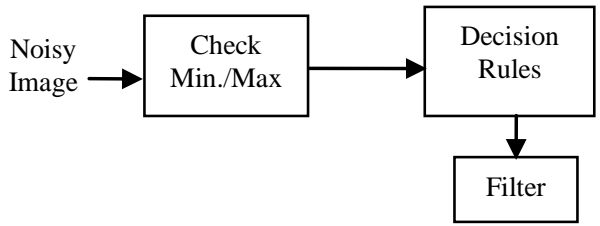

Fig.1 Impulse Detection

In this paper, impulse detection is used to detect the random valued impulse noise between minimum and maximum noise intensity range. The random valued impulse noise range for the shades of salt and pepper is fixed dynamically. In this paper, three different random valued impulse noise levels have been selected for experiment and are summarized in Table1.

\subsection{Proposed Filtering Technique}

Standard Median filtering scheme is subsequently used to remove impulse noise and preserve edge and fine details on digital images, depending on the characteristic of pixel. According to the decision-mechanism, impulse noise is identified within the filtering window. In this paper, filtering operation is obtained in two decision levels are as: 1) Action of "no filtering" is performed on the uncorrupted pixels at the first decision level. In second decision level, noisy pixels are removed while preserving of edges and fine details are preserved on the digital image simultaneously. This filtering operation is obtained by applying median filtering operation at the current pixel within the sliding window on digital image. These values are the impulse noise intensity values. If the current pixel is detected as an uncorrupted pixel and it is left unaltered, otherwise, it is corrupted. Then median filter is performed on it. In order to apply the proposed filter, the corrupted and uncorrupted pixels in the selected filtering window are separated and then numbers of uncorrupted pixels are determined. The corrupted pixels in the image are detected by checking the pixel element value in the dynamic range of maximum and minimum respectively. Median is calculated only for a number of uncorrupted pixels in selected filtering window. Then the corrupted pixel is replaced by this new median value. This condition is used to preserves the Edges and fine details of the given image. Consider an image of size $\mathrm{M} \times \mathrm{N}$ having 8-bit gray scale pixel resolution. The steps involved in detecting the presence of an impulse or not are described as follows:

Step 1) A two dimensional square filtering window of size $3 \mathrm{x}$ 3 is slid over on a contaminated image $x(i, j)$ from left to right, top to bottom in a raster scan fashion.

$$
w(i, j)=\left(X_{-n(i, j)}, \ldots, X_{-1(i, j)}, X_{0(i, j)}, X_{1(i, j)}, \ldots, X_{n_{(i, j)}}\right)
$$

where $X_{0(i, j)}$ (or) $X_{(i, j)}$ ) is the original central vector-valued pixel at location $(i, j)$. Impulse noise can appear because of a random bit error on a communication channel. The source images are corrupted only by random valued impulse noise in the dynamic range of shades of salt $\&$ pepper.

Step 2) In the given contaminated image, the central pixel inside the $3 \times 3$ window is checked whether it is corrupted or not. If the central pixel is identified as uncorrupted, it is left unaltered. A $3 \times 3$ filter window $w(i, j)$ centered around $X_{0(i, j)}$ is considered for filtering and is given by

$$
w(i, j)=\left(X_{-4(i, j)}, \ldots, X_{-1(i, j)}, X_{0(i, j)}, X_{1(i, j)}, \ldots, X_{4(i, j)}\right)
$$

Step 3) If the central pixel is identified as corrupted, determine the number of uncorrupted pixels in the selected filtering window and median value is found among these uncorrupted pixels. The corrupted pixel is replaced by this median value.

Step 4) Then the window is moved to form a new set of values, with the next pixel to be processed at the centre of the window. This process is repeated until the last image pixel is processed. Pictorial representation of this filtering procedure is shown in Fig.2. In Figure 2 image example matrix is corrupted with random valued impulse noise in the range of $1-9 \& 246-255$. In the present case, noisy pixel and noise free pixels are identified Uncorrupted pixels are arranged in ascending order and then median value (220) is determined from these pixels. The central pixel is replaced by median value within the filtering window on digital images. 


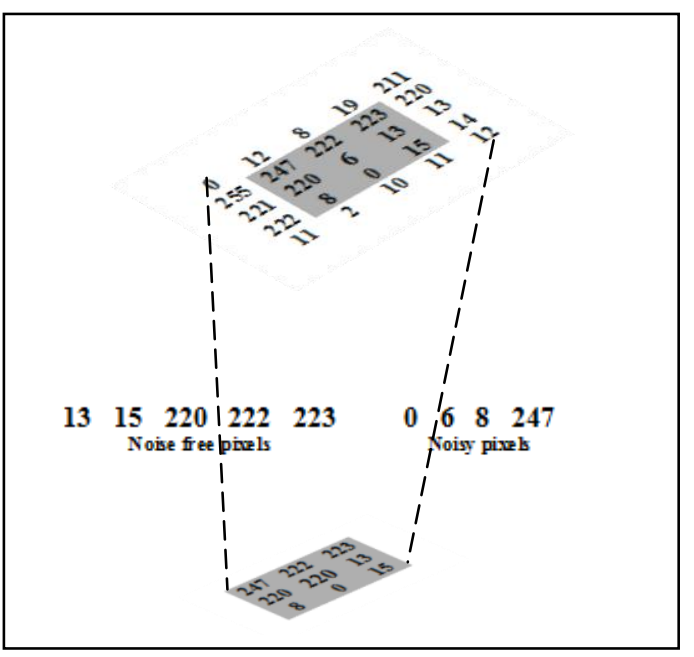

Fig.2 Pictorial representation of the proposed filter

This Impulse noise detection and filtering is based on the following condition:

$$
\begin{aligned}
& \text { if } X_{L N L}<X_{O(i, j)}<X_{H N L} \\
& \quad\left\{X_{O(i, j)}\right. \text { is a noiseless pixel; } \\
& \text { no filtering is performed on } \left.X_{O(i, j)}\right\} \\
& \quad X_{O(i, j)}=X_{O(i, j)} \\
& \text { else }
\end{aligned}
$$

$$
\left\{X_{O(i, j)}\right. \text { is a noisy pixel; }
$$

determine the median value\}

$$
X_{O(i, j)}=X_{m e d}
$$

end

where, $X_{0 i, j}$ is the intensity of central pixel inside the filtering window, $X_{L N L}, X_{H N L}$ and $X_{\text {med }}$ are the low noise level, higher noise level and median pixel value in filtering window of noisy image. The proposed filtering algorithm is lower complexity than Boundary discriminative noise filtering technique [17] and A Noise fading technique for images highly corrupted with Impulse Noise (NFT) [23] in terms of computational requirements. Further, the Boundary discriminative noise filtering technique considerably more complex in terms of computation therefore, it has not been taken into account for performance comparison with the proposed filtering algorithm. The proposed filter exhibits superior performance than the NFT in terms of eliminating fixed impulse noise up to $80 \%$ and preserving edges and fine details.

\section{RESULTS}

Experiment is presented which is used to characterize the proposed filter. The Filtering schemes are tested using the size of 3 X 3 windows with four different images of size $256 \mathrm{X}$ 256. In order to analyze the performance of the proposed filter approach, the performance evaluation factor Peak Signal to Noise Ratio (PSNR) is used. The objective measure, namely, Peak Signal to Noise Ratio (PSNR) is defined as follows:

$$
P S N R=10 \log _{10}\left[\frac{255 * 255}{M S E}\right]
$$

where

$$
M S E=\frac{\Sigma|X(i, j)-F(i, j)|^{2}}{\text { rowx } * \text { col }}
$$

where, $(i, j)$ denotes the number of rows and columns in the image data, $X(i, j)$ represents the pixel intensities of the original image at the position of $(\mathrm{i}, \mathrm{j}), F(i, j)$ represents the output intensities in the filtered image at the position of $(i, j)$. Fig.3 illustrate that the subjective performance on Lena image corrupted by noise density at $80 \%$ level.
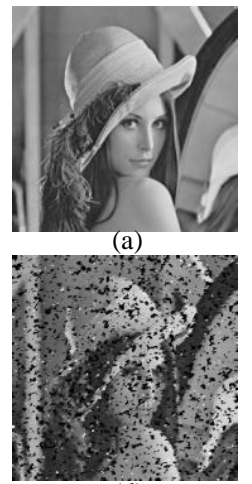

(d)

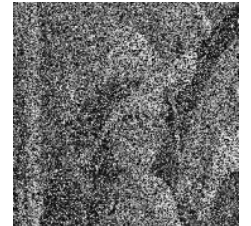

(b)
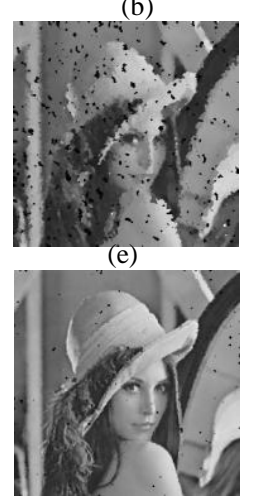

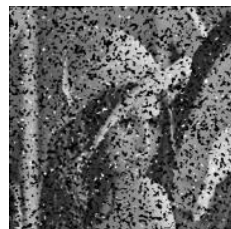

(c)

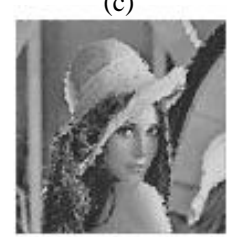

(f)
Fig.3 Performance of proposed filtering technique compared with other filtering techniques; (a) Noise free Lena image, (b) Image corrupted with $80 \%$ random valued impulse noise, (c) Image restored by MF (d) Image restored by WMF, ((e) Image restored by CWMF, (f) Image restored by NFT and (g) Image restored by Proposed filter.

The values of objective measures obtained by applying the proposed filter and compared with existing filtering techniques namely; Median filter (MF), Weighted median filter (WMF), Center weighted median filter (CWMF), Noise fading technique on Lena image contaminated with the impulse noise of various densities are summarized in Table 2 and Table 3. This is graphically illustrated in Fig.4.

The values of objective measures obtained by applying the proposed filter on four test images contaminated with the impulse noise of various is summarized in Table 4 and subjective evaluation is illustrated in Fig.5. This represents the different test images restored by the proposed filter is free from noise and are pleasant for visual perception. The proposed filter is noted to exhibit superior quantitative performance for noise densities up to $80 \%$ and good qualitative performance for noise densities up to $90 \%$.

Table 1. Experimented noise level

\begin{tabular}{|c|c|c|}
\hline \multirow{2}{*}{ S.No } & \multicolumn{2}{|c|}{ Noise range } \\
\cline { 2 - 3 } & Shades of Black & Shades of white \\
\hline 1. & $1-9$ & $246-255$ \\
\hline 2. & $1-19$ & $236-255$ \\
\hline 3. & $1-59$ & $196-255$ \\
\hline
\end{tabular}

These three noise ranges are experimented on Lena image for performance evaluation. 
Table 2. Performance of PSNR for different filtering techniques on Lena image for various densities noise

\begin{tabular}{|c|c|c|c|c|}
\hline & \multicolumn{4}{|c|}{$\begin{array}{c}\text { Noise Percentage } \\
\text { (salt \& pepper) }\end{array}$} \\
\hline $\begin{array}{c}\text { Filtering } \\
\text { Techniques }\end{array}$ & 20 & 40 & 60 & 80 \\
\hline MF & 22.62 & 18.85 & 15.01 & 12.33 \\
\hline WMF & 26.58 & 21.65 & 16.78 & 13.77 \\
\hline CWMF & 29.06 & 25.83 & 21.83 & 17.71 \\
\hline NFT & 35.15 & 29.63 & 27.22 & 21.95 \\
\hline Proposed Filter & 36.93 & 29.75 & 27.47 & 21.64 \\
\hline
\end{tabular}

Table 3. Performance of PSNR for BDND and the proposed technique on Lena image at various noise densities in the range of $(1-19,236-255)$

\begin{tabular}{|c|c|c|c|}
\hline & \multicolumn{3}{|c|}{ Noise Percentage } \\
\hline $\begin{array}{c}\text { Filtering } \\
\text { Techniques }\end{array}$ & 40 & 60 & 80 \\
\hline BDND & 23.19 & 21.97 & 18.87 \\
\hline $\begin{array}{c}\text { Proposed Filter } \\
\text { (noise range } \\
1-59,196-255)\end{array}$ & 28.11 & 23.90 & 19.50 \\
\hline
\end{tabular}

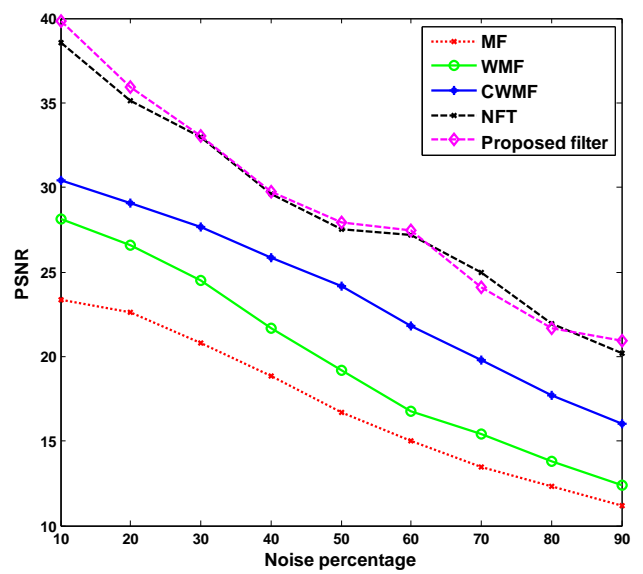

Fig.4 Performance of PSNR obtained by proposed filtering technique compared with other filtering techniques on Lena image corrupted by salt \& pepper

Table 4. Performance of PSNR for proposed filtering techniques on different test images for various densities noise in the range of $1-19 \& 236-255$

\begin{tabular}{|c|c|c|c|c|}
\hline & \multicolumn{4}{|c|}{ Noise Percentage } \\
\hline $\begin{array}{c}\text { Filtering } \\
\text { Techniques }\end{array}$ & 20 & 40 & 60 & 80 \\
\hline Lena & 35.93 & 29.07 & 24.29 & 20.29 \\
\hline Cameraman & 33.4853 & 27.9150 & 24.8636 & 20.8187 \\
\hline Baboon & 32.2428 & 27.0467 & 24.8022 & 20.6469 \\
\hline Pepper & 38.1902 & 33.2218 & 27.7815 & 22.9718 \\
\hline
\end{tabular}
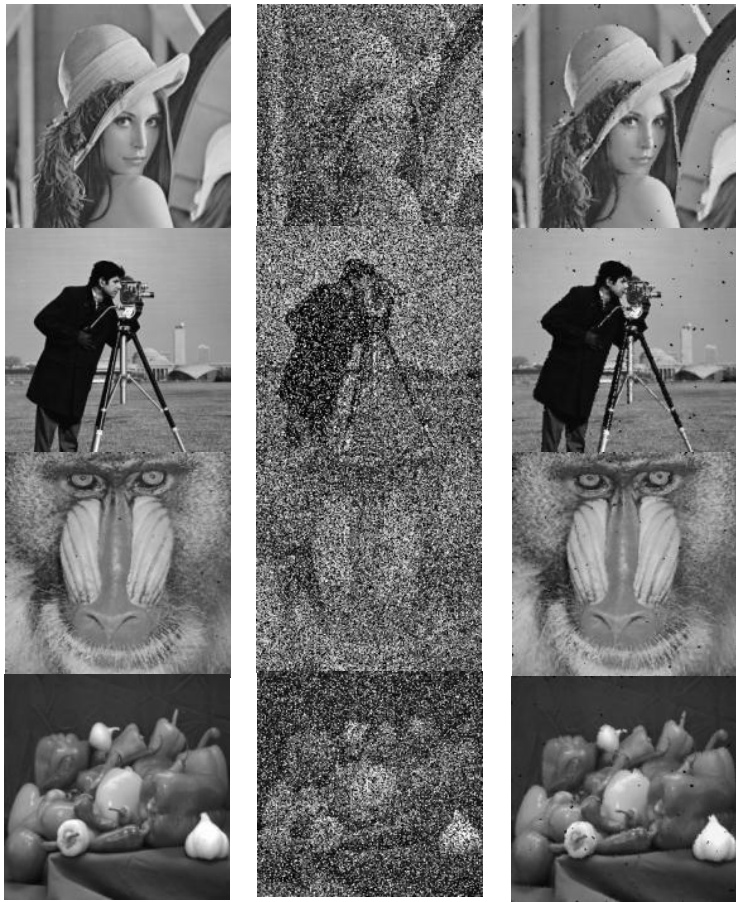

Fig.5 Subjective

performance illustration of proposed filtering technique for Lena, Cameraman, Baboon and Pepper images: noise free image in first column, images corrupted with $80 \%$ impulse noise in second column, Images restored by proposed Filtering Technique in third column.

Table 5. Experimented noise level on Lena image

\begin{tabular}{|c|c|c|c|}
\hline \multirow[b]{2}{*}{$\begin{array}{l}\text { Noise } \\
\text { level }\end{array}$} & \multicolumn{3}{|c|}{ Noise range } \\
\hline & $\begin{array}{l}\mathrm{M}=1-9 \& \\
\mathrm{n}=246-255\end{array}$ & $\begin{array}{l}\mathrm{M}=1-19 \& \\
\mathrm{n}=236-255\end{array}$ & $\begin{array}{l}M=1-59 \& \\
n=196-255\end{array}$ \\
\hline 5 & 43.41 & 42.66 & 33.95 \\
\hline 10 & 39.83 & 39.40 & 33.22 \\
\hline 20 & 35.51 & 34.88 & 31.63 \\
\hline 40 & 29.42 & 29.07 & 28.11 \\
\hline 60 & 24.43 & 24.29 & 23.90 \\
\hline 80 & 20.72 & 20.29 & 19.50 \\
\hline
\end{tabular}

There are three different densities of random valued impulse noise are experimented and is summarized in Table 5 for quantitative performance comparision.

\section{CONCLUSION}

The performance of proposed filtering scheme is quantitatively evaluated on different images using subjective and objective measures. With the proper decision rules, these filtering schemes offer improved visual quality of filtered images in addition to much reduced mean square error values. Simulation studies conducted by applying this filtering scheme on Lena test image contaminated with different levels of impulse noise show that the proposed filter is superior to that Noise fading technique (NFT) in terms of noise removal and fine details preservation. The main advantage of the proposed algorithm is reduction in computational complexity and it is fairly suitable for real-time image applications.

\section{REFERENCES}

[1] Pitas. I and Venetsanopou. A, “ Nonlinear Digital Filters : Principles and Application", Norwell, MA: Kluwer1990. 
[2] Astola.J and P.Kuosmanen, "Fundamentals of Nonlinear Digital Filtering", Boca Raton, FL: CRC, 1997.

[3] Brownrigg. D.R.K, "The weighted median filter", Commun Ass.Comput. Mach., Vol. 27, no. 8, pp. 807-818, Aug.1984

[4] Neal C Gallagher, "Theoretical Analysis of the Properties of Median Filters", IEEE Trans. on Acoustic, Speech and Signal Processing, Vol.ASSp.29, No.6, Dec., 1981

[5] GonzaloR.Arce, "A Generalization Weighted Median Filter Structure Admitting Real-valued Weights", IEEE Trans.on Signal Processing,Vol.22, No. 6, pp. 28492852,Dec. 1998

[6] Sung - Jea Ko and Yong Hoon Lee, "Center Weighted Median Filters and Their Applications to Image Enhancement", IEEE Trans.on Circuits and Systems, Vol.38, No.9, pp. 984-993, Sep., 1991.

[7] Ko. S. - J and Lee. S.J, " Center weighted median filters and their applications to image enchancement", IEEE Trans. Circuits Syst., Vol. 15, pp. 984-993, Sept.1991.

[8] Sun. T and Neuvo. Y, "Detail - preserving median based filters in image processing", Pattern Recognition Letter, Vol. 15, pp. 341-347, 1994.

[9] H.Hwang and R. A. Haddad, "Adaptive Median Filters: New Algorithms and results",IEEE Trans. Image Processing, vol.4, No.4, pp. 499-502, April 1995.

[10] Florencio.D and Schafer.R, "Decision-based median filter using local signal statistics", in Proc.SPIE Int. Symp. Visual Communications image Processing, Chicago, Sept.1994.

[11] Yang. R, Gabbouj. M, and Neuvo.Y " An efficient design method for optimal weighted median filtering", in IEEE Int. Symp. Circuits Systems (ISCAS'94), Chicago, IL, , Sept. 1994

[12] Yong Hoon Lee and A. Kassam, “ Generalized Median Filtering and Related Nonlinear Filtering Techniques", IEEE Trans. on Acoustic, Speech and Signal Processing,Vol. ASSp.33, No.3,June, 1985.

[13] Sun. T and Neuvo. Y, "Detail - preserving median based filters in image processing", Pattern Recognition Letter, Vol.15,pp. 341-347,1994.

[14] Zhou Wang and David Zhang "Progressive Switching Median Filter for the removal of Impulse Noise from Highly Corrupted Images", IEEE Trans. on Circuits and Systems- II: Analog and Digital Signal Processing, Vol.46, No.1, pp.78-80, Jan., 1999.
[15] Tao Chen and Kai - kuang Ma, "Tri - State Median Filter for Image Denoising",IEEE Trans. on Image Processing, Vol.8, No.12, pp.1834-1838, Dec., 1999.

[16] Shuqun Zhang and Mohammad A Karim, "A New Impulse Detector for Switching Median Filters", IEEE Signal Processing Lett., Vol.9, No.11, pp.360-363, Nov. 2002

[17] Pei - Eng Ng and Kai - Kuang ma, "A Switching Median Filter with Boundary Discriminative Noise Detection for Extremely Corrupted Images", IEEE Trans.on Image Processing, Vol.15, No.6, pp.1506-1516, June 2006

[18] R.Pushpavalli and E.Srinivasan, "Multiple Decision Based Switching Median Filtering for Eliminating Impulse Noise with Edge and Fine Detail preservation Properties", International conference on Signal Processing, CIT, Aug. 2007.

[19] R.Pushpavalli and E.Srinivasan, " Multiple Thresholds Switching Median Filtering for Eliminating Impulse Noise in Images",International conference on Signal Processing, CIT, Aug. 2007.

[20] M. Ciuc, V.Vrabie, M.Herbin, and C.Vertan, P. Vautrot, Adaptive Neighborhood best mean rank vector filter for impulsive noise removal," Proc. of IEEE ICIP, pp. 813-816, 2008

[21] R.Pushpavalli and E.Srinivasan, "Decision based Switching Median Filtering Technique for Image Denoising", CiiT International journal of Digital Image Processing, Vol.2, no.10, pp.405-410, Oct.2010.

[22] Hadi. Zayyani, Seyyedmajid. Valliollahzadeh, "Sparse Component Analysis (SCA) in Random-valued and Salt and Pepper Noise Removal", International conference on Electrical Engineering, May, 2008.

[23] Noritaka Y, Munenori O, Jianming L, Hiroo S and Takashi Y., "A Random-valued Impulse Noise Detector Using Level Detection", Circuits and Systems, 2005. ISCAS 2005. IEEE International Symposium on may 2005.1

[24] Hancheng $\mathrm{Yu}, \mathrm{Li}$ Zhao, and Haixian Wang, "An Efficient Procedure for Removing Random-Valued Impulse Noise in Images", on IEEE Signal Processing Letters, VOL. 15, 2008.

[25] S.Indu and Chaveli Ramesh, "A Noise Fading Technique for Images Highly Corrupted with Impulse Noise," Proceedings of the ICCTA07, IEEE.

[26] Pei-Eng Ng And Kai-Kuang Ma, "A Switching Median Filter With Boundary Discriminative Noise Detection For Extremely Corrupted Images", IEEE Transaction on Image processing, Vol15, No.6, pp 1506-1516, 2006. 